\title{
A 10 años de la emergencia sanitaria anestésico-quirúrgica en Uruguay. Comparación de los resultados de la oferta actual con la proyectada en 2011
}

10 years after the anesthetic-surgical health emergency in Uruguay. Comparison of the results of the current offer with that projected in 2011 10 anos após a emergência de saúde anestésico-cirúrgica no Uruguai. Comparação dos resultados da oferta atual com a projetada para 2011

Fiorella Cavalleri', Juan Pablo Bouchacourt², Beatriz Noya², Laura Illescas², Ana Rodríguez², Pablo Castromán², Juan Riva3

\section{Resumen}

Introducción: durante el 2011 se propuso un modelo de simulación para valorar la oferta, la relación demanda/necesidades y las tendencias en el tiempo de anestesiólogos y establecer escenarios posibles.

Objetivo: comparar la oferta actual de anestesiólogos con la proyectada por el modelo de simulación en el año 2011.

Material y métodos: se realizó una actualización a partir de las bases de datos disponibles. Se registró número de anestesiólogos titulados y no titulados, su distribución demográfica y se comparó con los escenarios propuestos en un estudio anterior.

Resultados: la tasa de médicos con competencias en anestesiología fue de 14,2/100.000 habitantes y la de anestesiólogos titulados 12/100.000. La distribución de edad presentó una mediana de 54 años, el $90 \%$ central se encontraba entre 36 y 75 años. El $57 \%$ correspondió a mujeres. 21,8 anestesistas/100.000 habitantes corresponden a Montevideo y área metropolitana contra $7 / 100.000$ en el resto del país. Hubo un incremento promedio del $50 \%$ en el número de plazas para la formación, un aumento de solicitudes de reválida ( 11 en el período estudiado) y un incremento del 17,5\% de la producción quirúrgica en el subsector público.

Conclusiones: el número de anestesiólogos disponibles en el año 2020 sugirió que la proyección realizada en dos de los escenarios propuestos en investigación anterior reprodujeron adecuadamente la oferta real. Se observó un crecimiento de la participación de mujeres, mayor porcentaje en franjas etarias menores, centralización de los recursos humanos en Montevideo y una edad de retiro mayor. La principal limitación sigue siendo contar con información oportuna y de calidad. Es fundamental la utilización de metodologías rigurosas y de aplicaión sistemática para que quienes planifican puedan tomar decisiones informadas.

Palabras clave: Anestesiología

Anestesiólogos

Key words: $\quad$ Anesthesiology

Recursos humanos

Anesthesiologists

Análisis estadístico

Workforce

Distribución de médicos

Statistical analysis

Physicians distribution

\footnotetext{
1. Profesora Adjunta del Departamento de Medicina Preventiva y Social. Licenciada en estadística.

2. Profesor/a Agregado/a de Anestesiología.

3. Profesor de Anestesiología.

Departamento de Anestesiología. Departamento de Medicina Preventiva y Social

Facultad de Medicina. Universidad de la República.

Correspondencia: Juan Riva. Hospital de Clínicas “Manuel Quintela”. Avenida Italia s/n. Piso 17. Correo electrónico: juanrivafortunato@gmail.com Estudio realizado con fondos del Departamento de Anestesiología. Facultad de Medicina. UdelaR.

Los autores declaran no tener conflictos de interés.

Recibido: 3/12/2020

Aprobado: 12/4/2021

Attribution-NonCommercial 4.0 International (CC BY-NC 4.0)
} 


\section{Introducción}

Durante la emergencia sanitaria en la asistencia anestésico-quirúrgica ${ }^{(1)}$ decretada en el año 2010, se desarrolló un método estadístico que permitió estimar las necesidades de anestesiólogos en el país, el cual fue una herramienta diagnóstica de gran utilidad ${ }^{(2)}$. De su análisis surgió la necesidad de realizar una proyección en el tiempo, para lo que los Departamentos de Métodos Cuantitativos y de Anestesiología de la Facultad de Medicina (UdelaR) propusieron un modelo de simulación que permitió, con los datos disponibles, valorar las tendencias en el tiempo y establecer escenarios posibles en un intervalo de tiempo dado ${ }^{(3)}$. En base a esto se sugirió cuál sería la trayectoria esperada y la mejor forma de cubrir la brecha en el mínimo tiempo posible, proyectando un incremento en la actividad quirúrgica. La conclusión fue que para los escenarios esperables en nuestro sistema de salud sería posible cerrar la brecha en el 2015. Sin embargo, contemplando el crecimiento de los procedimientos quirúrgicos, las necesidades de recursos humanos en anestesiología se aproximarían a 12,7 anestesiólogos cada 100.000 habitantes para el año 2025.

Un aspecto esencial en el potencial impacto que pueden tener estos modelos se basa en su seguimiento y auditoría lo cual permite modificarlo frente a situaciones cambiantes tanto en aspectos demográficos como en políticas de salud ${ }^{(4,5)}$. Sin embargo quedó en evidencia que aún modelos de relativa baja complejidad estructural, con número acotado de variables, implican un desafío en cuanto a disponibilidad de información de calidad para desarrollarlos y evaluarlos. Estas limitaciones hacen difícil evaluar el modelo en sí mismo, no obstante, el detenernos en un año específico y desde éste valorar si las proyecciones realizadas reprodujeron aproximadamente la realidad para ese año específico y para una serie de parámetros y supuestos, puede ser un aporte adicional al momento de diseñar estas políticas

El objetivo del presente estudio fue comparar la oferta de anestesiólogos en el 2020 con la proyectada por modelo de simulación en el año 2011.

\section{Métodos}

\section{Determinación de la oferta de anestesiólogos en el año 2020}

La información fue obtenida de las siguientes fuentes: se actualizó la base de datos que se elaboró para anteriores estudios ${ }^{(2,3)}$ en base a los registros del Departamento de Anestesiología de la Facultad de Medicina (UdelaR). El principal criterio utilizado para ello fue el de incluir personas que en 2020 estaban ejerciendo funciones de anestesita en el mercado laboral.
Frente a faltante de datos y/o dudas o inconsistencias se consultó en forma telefónica a cada uno de los profesionales. El número de procedimientos realizados en la Administración de los Servicios de Salud del Estado (ASSE) fueron extraídos del Sistema de Descripciones Operatorias.

Los datos registrados fueron los siguientes: número de médicos vinculados a la especialidad definidos como 1) número de anestesiólogos titulados de acuerdo a las exigencias de estudios, incluyendo las pruebas finales rendidas en el Departamento de Anestesiología; 2) número de médicos que finalizaron la etapa de formación y/o reválidas pero que aún no rindieron la prueba final; 3) número de residentes de anestesiología en formación y anestesiólogos extranjeros que cursan programa de reválida.

Se registró sexo, rango de edades y principal lugar de trabajo (Montevideo/Interior).

\section{Escenarios priorizados del modelo de simulación para realizar comparación con el año 2020}

Los escenarios propuestos en el estudio de simulación ${ }^{(3)}$ fueron los siguientes:

- Escenario 1: se consideró una demanda/necesidad de 11,28 anestesiólogos cada 100.000 habitantes y se estableció un número fijo de ingresos a la residencia (20 cargos) desde el 2013, independientemente de la brecha entre oferta y demanda/necesidad, la cual se proyectaba sería positiva a partir del 2015. Manteniendo este programa se generaría un superávit progresivo anual, hasta alcanzar un exceso relativo de 50 anestesiólogos a los diez años.

- Escenario 2: se consideró una demanda/necesidad estable con una reducción de la oferta anual que permitiría el cierre de la brecha para el 2015, estabilizando el número de especialistas en formación para mantener la brecha estable.

Se proyectó para el año 2020, en los dos escenarios considerados, una oferta de 419 y 392 anestesiólogos respectivamente.

La razón por la que se priorizaron estos escenarios fue que de implementarse los criterios en ellos establecidos permitirían llegar rápidamente a una oferta de anestesiólogos que permitiera satisfacer la demanda/necesidad de acuerdo a la estructura docente disponible para formar nuevos recursos humanos y que contemplara un incremento de la producción quirúrgica. Por otra parte permitiría crear por parte de la Facultad de Medicina (UdelaR) una estructura docente estable y económicamente sustentable. 
Tabla 1. Características de la población estudiada

\begin{tabular}{lcc}
\hline & $n(501)$ & $\%$ del total \\
\hline Anestesiólogos titulados & 422 & 84,2 \\
Médicos completaron su formación & 21 & 4,3 \\
Médicos en formación & 58 & 11,5 \\
Sexo (F/M) & $285 / 216$ & $57 / 43$ \\
Montevideo/Interior & $385 / 116$ & $77 / 23$ \\
Rango de edades (años) & & \\
$\quad$ Menores de 40 & 200 & 40,5 \\
$\quad$ Entre 41 y 60 & 208 & 42,1 \\
$\quad$ Más de 61 & 86 & 17,4 \\
\hline & \\
$\begin{array}{l}\text { Montevideo/Interior = lugar donde predominante ejerce la profe- } \\
\text { sión. Datos obtenidos de la base de datos del Departamento de } \\
\text { Anestesiología (UdelaR) }\end{array}$
\end{tabular}

\section{Comparación entre la oferta de anestesiólogos en el 2020 y la proyección realizada para este año en el 2011}

Se compararon los datos actuales (2020) con la proyección realizada por el modelo de simulación: tasa de anestesiólogos cada 100.000 habitantes, oferta anual de residentes y número en formación, total de anestesiólogos titulados y "brecha" definida por el número necesario para cubrir la demanda/necesidad planteada.

\section{Resultados}

En la tabla 1 se muestran los resultados en cuanto a las características del grupo estudiado. De acuerdo a las proyecciones del Instituto Nacional de Estadísticas ${ }^{(6)}$ en 2020 la población de Uruguay ascendió a 3.530 .912 habitantes. La tasa de médicos con diferentes grados de competencias en anestesiología fue de 14,2/100.000 habitantes y con título otorgado por la Escuela de Graduados 12/100.000 habitantes. Considerando la población de Montevideo y su área metropolitana de 1.764.745, la tasa de anestesiólogos para Montevideo fue de $21,8 / 100.000$ habitantes contra $7 / 100.000$ en el resto del país.

En la tabla 2 se muestra la distribución según sexo y radicación de acuerdo con la edad. El 76,8\% del total de los anestesiólogos se encuentran radicados en Montevideo. En el interior del país predominan hombres y mayores de 61 años. No hubo diferencia significativa en la proporción de hombres $(42,3 \%$ vs $45,7 \%)$ ni de mujeres (57,7\% vs 54,3\%) en Montevideo e interior del país respectivamente.
Tabla 2. Distribución por sexo y radicación según edad.

\begin{tabular}{lccc}
\hline & Menores de 40 & $41-60$ & 61 o más \\
\hline Sexo (F/M) & $113 / 87$ & $129 / 80$ & $39 / 47$ \\
Montevideo/Interior & $175 / 25$ & $249 / 69$ & $69 / 17$ \\
\hline & & \\
$n=501$. Datos obtenidos de la base de datos del Departamento de \\
Anestesiología (UdelaR).
\end{tabular}

Tabla 3. Número de ingresos de nuevos residentes por año.

\begin{tabular}{|lc|}
\hline 2020 & 18 \\
2019 & 12 \\
2018 & 12 \\
2017 & 17 \\
2016 & 16 \\
2015 & 19 \\
2014 & 14 \\
2013 & 20 \\
2012 & 23 \\
2011 & 32 \\
\hline Datos aportados por la Escuela de graduados (UdelaR). \\
\hline
\end{tabular}

En la tabla 3 se muestran los cupos anuales fijados por la Comisión Honoraria de Residencias Médicas. Varió desde un valor máximo de 32, el año en que se decretó la emergencia sanitaria, a 12 en los años 2019 y 2018 , con un promedio de 18 en el período estudiado. De acuerdo con los datos disponibles en la Escuela de Graduados, en el período correspondiente a 2000-2010 el promedio fue de 12 cupos, por lo que corresponde a un incremento del $50 \%$.

Durante el período 2004-2007 se registraron 40 solicitudes de intención de emigrar ${ }^{(7)}$, mientras que entre el 2010-2020 fueron postulados a nuestro Departamento por parte de la Escuela de Graduados 11 solicitudes de reválida. Cinco de ellas accedieron al título habilitante como médicos anestesiólogos, cinco están en condiciones de presentarse a la prueba final y uno no completó el proceso.

En la tabla 4 se muestran los procedimientos realizados durante el período 2014-2019 en ASSE donde se registró un aumento del 17,5\% de los que requieren la presencia de un anestesiólogo. 
Tabla 4. Número de procedimientos quirúrgicos con anestesista por año en ASSE.

\begin{tabular}{lcccccc}
\hline & 2014 & 2015 & 2016 & 2017 & 2018 & 2019 \\
\hline En BQ coordinadas & 19.490 & 21.318 & 23.144 & 25.476 & 24.449 & 22.925 \\
En BQ urgencias/emergencia & 20.757 & 20.851 & 20.868 & 20.682 & 20.799 & 21.142 \\
Fuera de BQ coordinadas & 527 & 839 & 2.649 & 2.745 & 3.483 & 3.816 \\
Fuera de BQ urgencias/emergencia & 10 & 21 & 47 & 39 & 91 & 70 \\
Total & 40.784 & 43.029 & 46.708 & 48.942 & 48.822 & 47.953 \\
\hline
\end{tabular}

Datos extraidos del Sistema de Descripciones Operatorias de ASSE. BQ: block quirúrgico.

\section{Discusión}

El modelo de simulación ${ }^{(3)}$ permitió proyectar la oferta, la relación demanda/necesidad y los equilibrios y desequilibrios entre ambas, a partir de una serie de parámetros y condiciones que determinaron ciertas trayectorias para los distintos escenarios considerados. El aporte de los distintos escenarios fue servir de insumo para comprender la dinámica bajo diferentes condiciones y posteriormente, en función de una trayectoria esperada, tomar decisiones acerca de los parámetros sobre los que se podía incidir. Con la información del total de anestesiólogos para 2020 se evidenció que la oferta fue aproximadamente la que se proyectó en los escenarios considerados. Lo importante no fue que la realidad se aproximó a lo que se proyectó como resultado en sí mismo, sino como la síntesis de la comprensión de un problema complejo, para el que se simularon distintas trayectorias a través de un modelo, sujeto a ciertas restricciones, seguidas de medidas concretas sobre ciertos parámetros, en función de un objetivo al cual aproximarse.

Para 2020 se proyectaron, en el escenario 1 (20 plazas anuales de formación) un total de 419 anestesiólogos titulados, mientras que en el escenario 2 (reducción progresiva de nuevas plazas de formación) se proyectaron 392. El número actual correspondió a 422 anestesiólogos titulados y 501 en total. Esta diferencia relativa puede ser explicada, en primer lugar, porque los llamados a nuevos cargos de residentes de anestesiología presentaron variaciones respecto a los escenarios planteados. Las decisiones adoptadas estuvieron más próximas a las propuestas en el escenario 2, aunque la reducción progresiva del número de cargos fue menos acentuada, ya que se llamaron 18 cargos por año. El Departamento de Anestesiología sugirió mantener una política de plazas fijas ya que la brecha positiva generada tomaba en cuenta un incremento en la actividad anestésica ${ }^{(3)}$. No obstante, la decisión en cada llamado también depende de la posibilidad de financiación que ofrecen las instituciones a la Comisión Honoraria de Residencias Médicas. Nuestro país no es ajeno a lo que sucede en otros medios. La disminución de anestesiólogos registrada en Estados Unidos durante los años 90 tuvo como causa principal un deterioro en la financiación de los nuevos cargos de formación ${ }^{(8)}$. En más de 70 instituciones durante el período 2000-2006 se constató una disminución continua del 5\%. La medida que se propuso para revertir esta situación fue asegurar un apoyo institucional continuo para mantener financieramente solventes a los departamentos donde se forman recursos humanos en anestesiología $^{(9)}$. En segundo lugar, hubo un cambio significativo en la tendencia migratoria que afectó tanto la oferta de profesionales como de recursos en formación. Mientras que en el período 2004-2007 se registraron 40 solicitudes de intención de emigrar, superando el número de títulos otorgados en ese período ${ }^{(7)}$, durante el período 2010-2020 no sólo se registró el retorno de un número considerable de profesionales (especialmente de Chile y España), sino que 11 anestesiólogos extranjeros solicitaron la reválida para ejercer en nuestro medio. En tercer lugar, estimamos una edad de retiro a los 67 años ${ }^{(3)}$. Por las características de nuestro sistema, un número importante de anestesiólogos se retiran sólo parcialmente a esa edad, situación que se da en otros medios y que deberá ser estudiada de otra forma para la estimación de recursos humanos ${ }^{(4)}$.

Un segundo hallazgo fue que, partiendo de una tasa de anestesiólogos titulados de 11,8 cada 100.000 habitantes (2011), la tasa actual es de 12. Estas cifras son comparables a otros medios como Reino Unido (10), Suiza $(14,4)$, Francia $(14,8)^{(10)}$ e Israel $(10)^{(11)}$. Mientras que nuestro análisis incluyó solamente anestesiólogos titulados, los países previamente mencionados incorporaron "administradores de anestesia" (residentes de anestesia, nurses anestesistas y médicos habilitados a 
realizar algún tipo de procedimientos). Por esta razón, hicimos adicionalmente el ejercicio de incorporar en la oferta del 2020 a los residentes de anestesiología, ya que participan activamente en la asistencia anestesiológica, especialmente en los servicios de ASSE. De esta forma, la tasa en Uruguay ascendió a 14,2/100.000 habitantes. Por otra parte, debe considerarse que las competencias en Uruguay están limitadas a la anestesiología mientras que en parte de los países antes mencionados la formación es común a la medicina intensiva ${ }^{(5)} \mathrm{y}$ debe proveer recursos humanos para ambas especialidades. Alemania posee una tasa de anestesiólogos de 19,9/100.000 habitantes, pero sus competencias incluyen los servicios de emergencia prehospitalaria ${ }^{(10)}$.

Con respecto a la distribución demográfica, constatamos que persiste una importante centralización de los anestesiólogos en Montevideo (76,8\%), similar a lo reportado en estudios anteriores ${ }^{(12)}$. Analizando el número de anestesiólogos según población, podemos evidenciar una importante desigualdad en la distribución, contando Montevideo y el área metropolitana con 21 anestesiólogos cada 100.000 habitantes mientras que el interior dispone de $7 / 100.000$. A esto debe sumarse un mayor porcentaje de anestesiólogos en las franjas etarias superiores.

Los estudios previos en anestesiología mostraron una tendencia a una mayor participación de las mujeres en la especialidad ${ }^{(3,12)}$. En 2010, un 54\% del total de los anestesiólogos fueron mujeres, siendo el porcentaje actual de $59 \%$, el cual alcanza el $61,7 \%$ cuando se analiza el grupo etario entre 40 y 60 años. Estas cifras son superiores a otros países, donde los porcentajes se ubicaron en el entorno del $30 \%{ }^{(5,11)}$. Existen datos controversiales con respecto a la distribución del trabajo de acuerdo al género, en una misma revisión se señala por una parte que la proporción de mujeres que trabajan a tiempo completo es similar a la de los hombres, por otra, una encuesta muestra que sólo el $27 \%$ trabaja a tiempo completo ${ }^{(5)}$. Estos últimos datos son coincidentes con Schubert y colaboradores ${ }^{(4)}$, que señalan un predominio del trabajo parcial en las mujeres por lo que asumen una reducción del 10\% en su contribución al trabajo en anestesiología.

Previo a 2010 las franjas etarias estaban distribuidas en tercios iguales, lo cual se consideró un llamado de atención ya que el tercio de mayor edad sería, por un lado, menos propenso a aumentar la carga laboral y, por otro, existía la posibilidad de retiro de un número importante de anestesiólogos en corto tiempo ${ }^{(12)}$. El registro actual muestra un predominio significativo de los dos tercios de menor edad, que da cuenta de un perfil demográfico menos envejecido.

La demanda de anestesiólogos está fuertemente influenciada por el volumen de cirugías. Las horas necesa- rias para el ejercicio de la anestesiología se estimaron de acuerdo al número de cirugías propuestas que deberían haberse realizado durante $2010^{(2)}$. De acuerdo con el registro actual, y considerando los servicios de ASSE, hubo un incremento del orden del $17 \%$ de los procedimientos que requieren anestesia entre los años 2014 y 2019. Esta cifra es comparable a la propuesta en nuestro estudio $^{(3)}$ como probabilidad de aumento de estos requerimientos y fue extrapolada de otros medios ${ }^{(10)}$ donde se estimaba que sería del orden del $1,5 \%$ a $2 \%$ anual. $\mathrm{Si}$ bien es un dato esencial, sería necesario incorporar otros, como por ejemplo la relación de cirugías con internación y ambulatorias y cirugías en pacientes ancianos, que serían determinantes en cuanto a la estructura necesaria. De acuerdo con las cifras del INE ${ }^{(6)}$ el incremento de la población superior a los 65 años se acompañó de un aumento aún mayor de las cirugías en esta franja etaria, especialmente en las vinculadas al Fondo Nacional de Recursos ${ }^{(13)}$.

Existen importantes limitaciones en nuestro sistema de salud que deben ser consideradas. La principal refiere a la accesibilidad a información de calidad. Estos datos surgen de diferentes fuentes a las que se accede por diferentes vías y mediante un proceso artesanal y engorroso. Esto determina una importante dificultad para incorporar nuevos elementos necesarios para la planificación de recursos humanos. Schubert y colaboradores ${ }^{(4)}$, en una actualización de un estudio sobre recursos humanos en Estados Unidos (1993-2001), sugieren la incorporación de género y especialmente su distribución en el trabajo de anestesiología, edad de retiro y su impacto en el trabajo parcial, efecto de la participación de no médicos, producción quirúrgica y su característica (edad de los pacientes, cirugía ambulatoria/no ambulatoria) y distribución en el sector público/privado. Concluyen entonces que un análisis significativo de la fuerza laboral en anestesia debe basarse menos en datos históricos y más en información que refleje el entorno de atención médica en constante cambio. El Centre for Workforce Intelligence $^{(5)}$ establece escenarios posibles, con una metodología dentro de la cual incorporan los sistemas dinámicos para el cálculo de la oferta/demanda de anestesiólogos. Con ello pretenden planificar a largo plazo la fuerza laboral, apoyar a quienes toman las decisiones y crear conciencia de los riesgos con respecto a las necesidades de recursos humanos. Sus conclusiones son aplicables a nuestro medio: 1) sugiere continuar cubriendo el número de puestos de formación de la especialidad para minimizar el riesgo de escasez a corto plazo. Nuestra dependencia de la propuesta voluntaria de las plazas de formación por parte de Instituciones públicas y privadas es una limitante mayor para cualquier planificación futura. 2) Se debe realizar un inventario cada 2 a 3 años, para lo 
cual se requiere información confiable y accesible, limitante importante que surge de este estudio. 3) Considerar la posibilidad de combinar las habilidades clínicas para ayudar a manejar el creciente rol perioperatorio del anestesiólogo. En este sentido los anestesiólogos en formación deben ser considerados y regulados como parte de la fuerza de trabajo y por tanto ajustar sus condiciones de trabajo a esta situación.

Una segunda limitación es la modalidad del trabajo en anestesiología. Existe una importante diversidad en cuanto a la forma de relacionamiento laboral, así como del modelo de trabajo que impide establecer escenarios más allá del número.

\section{Conclusiones}

El número de anestesiólogos para el 2020 se ajustó a lo esperado de acuerdo a las trayectorias planteadas y medidas adoptadas.

Se evidenció un crecimiento de la participación de mujeres en la especialidad y un mayor porcentaje de anestesiólogos en franjas etarias menores. Estos aspectos, junto a una persistente centralización de los recursos humanos en Montevideo y a una edad de retiro mayor a la propuesta son aspectos que deben ser incorporados en la planificación de los recursos humanos. Las principales limitaciones que hallamos fueron el acceso a información confiable que permita comparar con datos históricos e incorporar otras variables relevantes y así como la diversidad en la forma de trabajo de los anestesiólogos.

Una oferta insuficiente de anestesiólogos será causa fundamental de un deterioro significativo en cantidad y calidad de los recursos en la academia ya que se pierden espacios docentes y de investigación.

Un enfoque óptimo requeriría del establecimiento de metodologías rigurosas y de aplicación sistemática que permitan analizar la evolución de la fuerza de trabajo y proporcionar a quienes planifican y toman decisiones en salud, a quienes forman recursos humanos sanitarios, evidencia basada en proyecciones de oferta y deman$\mathrm{da} /$ necesidad para indicar la trayectoria del sistema bajo diferentes escenarios. Sería deseable evaluar el impacto de distintas intervenciones como el incremento de cupos de formación, tiempo de duración de la formación, variaciones en la edad de jubilación, carga horaria semanal, entre otros.

En la ley 19.301, referente al Sistema Nacional de Residencias Médicas ${ }^{(14)}$, se creó el Consejo Administrador Honorario que establece que el mismo fijará el número de cargos de Médicos Residentes así como la distribución cuantitativa de ellos. En tal sentido, es prioritario que dicho órgano cuente con metodologías aplicadas con rigor científico e información de calidad para tomar decisiones de manera informada.

\section{Summary}

Introduction: during 2011 a simulation method was proposed to assess the needs and trends over time of anesthesiologists and establish possible scenarios.

Objective. compare the current situation with the projections formulated in 2011 by the simulation method to evaluate anesthesiologists' supply and demand.

Material and methods: an update of the available databases was revised. The number and demographic distribution of the anesthesiologist workforce were recorded and compared with the proposed scenarios.

Results: the number of physicians with anesthesiology skills was 14.2/100.000, and wholly trained anesthesiologists were 12/100.000. The age distribution presented a median of 54 years, the central $90 \%$ were between 36 and 75 years old. $57 \%$ of the anesthesiologist are females. 21.8 workforce in anesthesiology/100.000 corresponds to Montevideo and the metropolitan area, against $7 / 100.000$ in the rest of the country. There was an average increase of $50 \%$ in the number of positions for training, an increase in revalidation applications, and a rise of $17.5 \%$ in a surgical procedure in the public subsector.

Conclusions: the prediction of the number of anesthesiologists and their characteristics for 2020 coincided with two of the proposed scenarios.

We verify a growth in female participation, a higher percentage in younger age groups, centralization of human resources in Montevideo and higher retirement age. The main limitation was access to reliable information.

\section{Resumo}

Introdução: durante 2011, foi proposto um modelo de simulação para avaliar a oferta, demanda / necessidades, tendências ao longo do tempo dos anestesiologistas e estabelecer possíveis cenários.

Objetivo: comparar a oferta atual de anestesiologistas com a projetada pelo modelo de simulação em 2011.

Material e métodos: foi feita uma atualização das bases de dados disponíveis. O número de anestesiologistas licenciados e não certificados e sua distribuição demográfica foram registrados e comparados com os cenários propostos em um estudo anterior.

Resultados: a taxa de médicos com habilidades em anestesiologia foi de 14,2/100.000 habitantes e a de anestesiologistas licenciados de 12/100.000. A distribuição de idade apresentou mediana de 54 anos, os centrais $90 \%$ estavam entre 36 e 75 anos. 57\% correspondiam a mulheres. 21,8 anestesistas / 100.000 habitantes correspondem a Montevidéu e região metropolitana 
contra 7 / 100.000 no resto do país. Houve um aumento médio de $50 \%$ no número de vagas para treinamentos, um aumento nas solicitações de revalidação (11 no período estudado) e um aumento de $17,5 \%$ na produção cirúrgica no subsetor público.

Conclusões: o número de anestesiologistas disponíveis em 2020 sugeria que a projeção feita em dos cenários propostos em pesquisas anteriores reproduzia adequadamente a oferta real.

Houve crescimento da participação feminina, maior percentual nas faixas etárias mais jovens, centralização dos recursos humanos em Montevidéu e maior idade de aposentadoria. A principal limitação continua sendo a disponibilidade de informaçao oportuna e de qualidade.

$\mathrm{O}$ uso de metodologias rigorosas e sistematicamente aplicadas é essencial para que aqueles que planejam possam tomar decisões informadas.

\section{Bibliografía}

1. Ley 18.711. Emergencia sanitaria de la asistencia anestésico quirúrgica. Montevideo, 30 de diciembre de 2010. Disponible en: https://legislativo.parlamento.gub.uy/temporales/leytemp5349551.htm [Consulta: 27 noviembre 2020].

2. Uruguay. Ministerio de Salud Pública. Sistema Nacional Integrado de Salud. División RRHH. Estimación de las necesidades de anestesistas. Montevideo, 2012. Disponible en: www.msp.gub.uy/andocasociado.aspx?7072,23955.[Consulta: 24 setiembre 2020].

3. Cavalleri F, Castromán P, Rodríguez A, Riva J. Utilidad de un método estadístico de simulación para evaluar la oferta y demanda de anestesiólogos en Uruguay (2011-2025). Propuesta para prospección y planificación de necesidad de recursos humanos en Anestesiología. Rev Méd Urug 2013; 29(3):165-73.

4. Schubert A, Eckhout G, Tremper K. An updated view of the national anesthesia personnel shortfall. Anesth Analg 2003; 96(1):207-14.
5. Centre for Workforce Intelligence. Review of the anaesthetics and intensive care medicine workforce. London, 2015. Disponible en: https://www.gov.uk/government/publications/review-of-the-anaesthetics-and-intensive-care-medicine-workforce[Consulta: 2 noviembre 2020].

6. Instituto Nacional de Estadística. Estimaciones y proyecciones de población. Montevideo, 2013. Disponible en: https://www.ine.gub.uy/estimaciones-y-proyecciones.[Consulta: 3 setiembre 2020].

7. Frau A. Informe sobre impacto en los egresos de las políticas de admisión y la tendencia emigratoria en especialidades quirúrgicas. Montevideo: UdelaR. Facultad de Medicina. Escuela de Graduados, 2009. Exp. № 070649-000320-09.

8. Grogono AW. National resident matching program results for 2005: slight increase in recruitment. ASA Newsl 2005; 69(5):20-33

9. Kheterpal S, Tremper KK, Shanks A, Morris M. Six-year follow-up on work force and finances of the United States anesthesiology training programs: 2000 to 2006. Anesth Analg 2009; 108(1):263-72.

10. Egger Halbeis CB, Macario A. Factors affecting supply and demand of anesthesiologists in Western Europe. Curr Opin Anaesthesiol 2006;19(2):207-12.

11. Weissman C, Eidelman LA, Pizov R, Matot I, Klein N, Cohn R. The Israeli anesthesiology physician workforce. Isr Med Assoc J 2006; 8(4):255-9.

12. Katzenstein Berro J, Saralegui Coli J, Ayala Cauterucci W. Situación laboral de la anestesiología en Uruguay: percepción de los anestesiólogos. Análisis y propuestas. Anest Analg Reanim 2010; 23(2):40-50.

13. Fondo Nacional de Recursos. Estadísticas e Informes. Disponible en: http://www.fnr.gub.uy/estadisticas [Consulta: 18 agosto 2020].

14. Ley 19.301. Régimen de residencias medicas hospitalarias. Montevideo, 8 de enero de 2015. Disponible en: https://www. impo.com.uy/bases/leyes/19301-2014 [Consulta: 3 setiembre 2020].

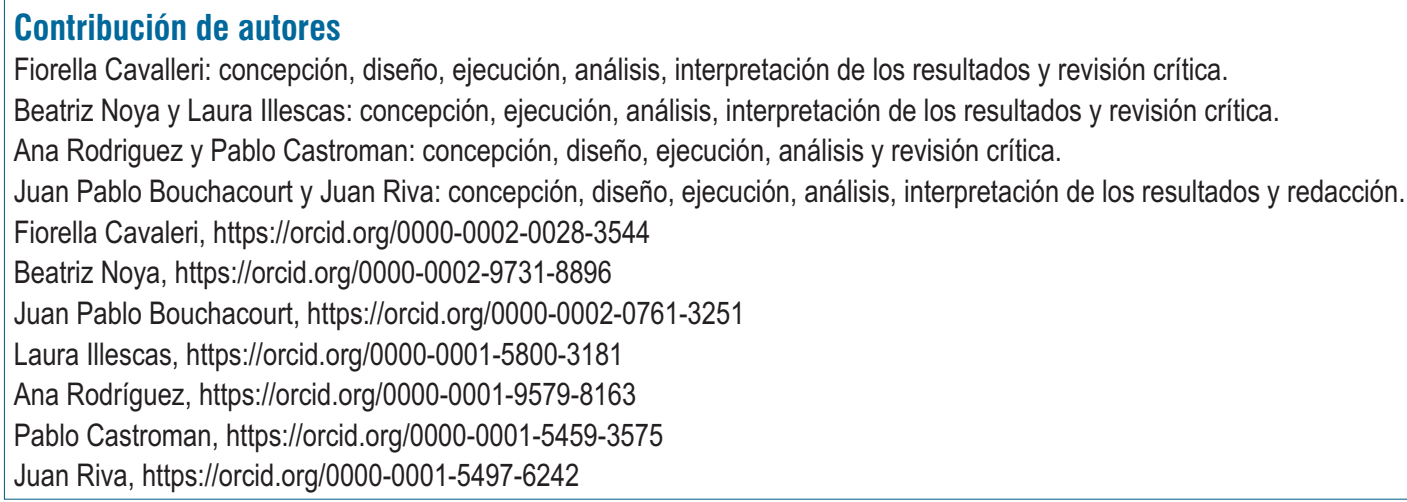

